SUBJECT AREAS:

SYNTHESIS AND

PROCESSING

SOLAR CELLS

Received

19 December 2013

Accepted

9 May 2014

Published

28 May 2014

Correspondence and requests for materials should be addressed to

A.G. (agupta@mint. va.edu) or N.B.

(nzhbao@njtech.edu.

\section{Monodisperse $\mathrm{Cu}_{2} \mathrm{ZnSnS}_{4}$ Nanocrystals with Controlled Shape and Structure}

\author{
Xiaoyan Zhang', Guobiao Guo', Cheng Ji', Kai Huang', Chenyang Zha', Yifeng Wang', Liming Shen',
} Arunava Gupta² \& Ningzhong Bao'

\footnotetext{
${ }^{1}$ State Key Laboratory of Materials-Oriented Chemical Engineering, Nanjing Tech University, Nanjing 210009, P. R. China,

${ }^{2}$ Centre for Materials for Information Technology, University of Alabama, Tuscaloosa, AL 35487, United States.
}

Monodisperse $\mathrm{Cu}_{2} \mathrm{ZnSnS}_{4}$ (CZTS) nanocrystals with tunable shape, crystalline phase, and composition are synthesized by efficient thermolysis of a single source precursor of mixed metal-oleate complexes in hot organic solvents with dissolved sulfur sources. Suitable tuning of the synthetic conditions and the $\mathrm{Cu} / \mathrm{Zn}+$ $\mathrm{Sn})$ ratio of the precursor has enabled precise control of the crystalline phase in the form of kesterite, or a newly observed wurtzite structure. Nanocrystals with morphology in the form of spherical, rice-like, or rod-like shapes are obtained over a wide range of compositions $(0.5 \leq \mathrm{Cu} /(\mathrm{Zn}+\mathrm{Sn}) \leq 1.2)$. Both the final products and intermediates for each shape exhibit consistent composition and structure, indicating homogenous nucleation and growth of single-phase nanocrystals. Thin films prepared from colloidal nanocrystal suspensions display interesting shape-dependent photoresponse behavior under white light illumination from a solar simulator.

\section{T}

hin film solar cells fabricated using colloidal semiconductor nanocrystals represent a promising low-cost approach to reducing the fabrication cost of solar cells ${ }^{1-3}$. The size, shape, crystal phase, and composition of the nanocrystals play an important role in determining the performance of solar conversion devices ${ }^{4-6}$. $\mathrm{Cu}_{2} \mathrm{ZnSnS}(\mathrm{Se})_{4}(\mathrm{CZTS}(\mathrm{Se}))$ compositions with kesterite structure have recently attracted a great deal of attention as novel alternative solar cell materials with suitable direct band gap $(\sim 1.4-1.5 \mathrm{eV})$, large optical absorption coefficient $\left(10^{4}-10^{5} \mathrm{~cm}^{-1}\right)$, and having non-toxic and earth-abundant constituents ${ }^{7-12}$. Single-junction solar cells fabricated using polycrystalline CZTS(Se) thin films as an absorber layer have achieved solar energy conversion efficiencies as high as $12.6 \%{ }^{7}$. Alternative approaches combining scalable solution processes, such as spray coating, spin-casting, and roll-to-roll printing, to fabricate thin film nanocrystal solar cells using inks of colloidal CZTS(Se) nanocrystals can further reduce the fabrication $\operatorname{cost}^{9-12}$. For example, a robust thin film coating method with composition tunability of CZTS(Se) thin films using kesterite-structured spherical nanocrystal suspensions has provided a cell efficiency of $7.2 \%{ }^{9}$. Recently, annealing mixed sulfide nanoparticles with variable ratios has enabled formation of kesterite-structured CZTSSe films with tunable chemical composition, and thus yielded an even higher efficiency of $8.5 \%{ }^{10}$. As for the crystal structure, the random distribution of A and B ions in the recently reported wurtzite phase chalcogenides offers flexibility for stoichiometry control, which is advantageous for fabrication of photovoltaics since it provides the ability to tune the Fermi energy over a wide range ${ }^{13,14}$. Thus, much effort has been devoted to the development and application of wurtzite-phase nanomaterials, such as $\mathrm{CuInS}_{2}{ }^{13-15}, \mathrm{CuIn}_{\mathrm{x}} \mathrm{Ga}_{1-\mathrm{x}} \mathrm{S}_{2}{ }^{16}, \mathrm{Cu}_{2} \mathrm{SnSe}_{3}{ }^{17}, \mathrm{CuFeS}_{2}{ }^{18}, \mathrm{Cu}_{2} \mathrm{CdSnSe}_{4}{ }^{19}$, etc. There are only a few reports on the synthesis of wurtzite phase CZTS nanocrystals ${ }^{20-22}$. Moreover, the synthesis of ternary and quaternary chalcogenide nanocrystals remains a challenge because of the difficulty in controlling the morphology, phase structure, and stoichiometry.

One of the more successful routes for the synthesis of chalcogenide nanocrystals involves the thermal decomposition of organometallic compounds, such as metal acetylacetonates ${ }^{11,23,24}$, metal carbonyls ${ }^{25-27}$, etc., in high boiling point solvents by hot-injection in the presence of surfactants. This procedure has been utilized for the formation of a variety of monodisperse binary- and ternary- chalcogenide nanoparticles with good crystallinity, uniform size, and simple shapes ${ }^{23-27}$. Recently, inexpensive, environmental-friendly metal-oleate complexes have been developed for the thermolytic synthesis of monodisperse oxides ${ }^{28-31}$ and chalcogenides ${ }^{32-35}$. However, synthesis of chalcogenide nanocrystals using metal-oleate complexes has thus far been limited to binary and 
ternary copper-indium-sulfide nanocrystals ${ }^{34}$ and heterostructures ${ }^{35}$. Herein, we report a simple, highly efficient synthesis process for producing monodisperse single-phase quaternary CZTS nanocrystals by injection of a novel single source precursor of homogeneously mixed metal-oleate complexes into hot solvents with dissolved sulfur source(s). Appropriate choice of the reaction temperature, higher than the decomposition temperature of constituent oleates, is critical for the compositional and structural uniformity of the nanocrystals (see Supplementary Fig. S1 online). The stoichiometry of the CZTS nanocrystals can be tuned over a wide composition range $(0.5 \leq \mathrm{Cu} /$ $(\mathrm{Zn}+\mathrm{Sn}) \leq 1.2)$ by simply varying the $\mathrm{Cu} /(\mathrm{Zn}+\mathrm{Sn})$ ratio of the metal-oleate complex precursor. Tuning the reaction temperature, the nature of solvents including oleylamine (OLA), 1-octadecene $(\mathrm{ODE})$, and oleic acid (OA), and sulfur reactants including elemental sulfur (S) and dodecanethiol (Dod-SH), has further enabled us to both tailor the morphology in the form of sphere, rod, or rice-like shape and control the crystal phase in the form of the kesterite or wurtzite structure.

\section{Results}

The morphology of the as-synthesized nanocrystals has been investigated using transmission electron microscopy (TEM). As shown in Figure 1, monodisperse CZTS nanocrystals in the form of spherical (Figure 1a, d), rod-like (Figure 1b, e), and rice-like (Figure 1c, f) shape are obtained depending on the synthesis conditions. Figure 1a displays the TEM image of spherical CZTS nanocrystals synthesized in a mixture of OLA and ODE. The average size of the nanocrystals is $12.3 \pm 0.6 \mathrm{~nm}$. In contrast, rod-like CZTS nanocrystals (Figure 1b) with an average length of $45.4 \pm 3.5 \mathrm{~nm}$ and diameter of $12.6 \pm 2.3 \mathrm{~nm}$ are synthesized in ODE. The use of $25 \mathrm{ml} \mathrm{OA}$ as a solvent leads to the formation of rice-like CZTS nanocrystals (Figure 1c), with average dimensions of $10.2 \pm 2.8 \mathrm{~nm}$ in length and $8.4 \pm 2.2 \mathrm{~nm}$ in maximum diameter. High-resolution (HR)-TEM images of individual nanocrystals for the three different shapes show clear lattice fringes, indicating the single crystalline nature of the nanocrystals. The $d$ spacing measured for 1D rod-like (Figure 1e) and rice-like (Figure 1f) nanocrystals are $0.314 \pm 0.006$ and $0.318 \pm$ $0.005 \mathrm{~nm}$, respectively, corresponding to the (002) lattice plane of wurtzite CZTS, indicating preferential growth along the [001] direction.

Unlike binary and ternary chalcogenides, quaternary CZTS offers more flexible physical properties because of the increased chemical and structural degrees of freedom ${ }^{7-12}$. However, this freedom also creates a challenge in controlling the stoichiometry and crystal structure. CZTS usually crystallizes in the kesterite or stannite structure. Recently, a new wurtzite crystal structure has been reported ${ }^{20-22}$. We have successfully synthesized CZTS nanocrystals with different crystal structure by varying the type of sulfur source and/or the reaction temperature. The use of Dod-SH has been considered to be a primary factor for the formation of wurtzite $\mathrm{CZTS}^{20}$ due to its strong coordination with metal cations. Wurtzite CZTS nanorods have been synthesized using a mixture of thiols with ODE as solvent ${ }^{21}$. In the present study, we found that the synthesis in ODE with the use of excess Dod-SH (40 times that of $\left.\mathrm{Cu}_{2} \mathrm{ZnSn}\left(\mathrm{C}_{18} \mathrm{H}_{33} \mathrm{O}_{2}\right)_{10}\right)$ results in the formation of wurtzite and kesterite structure at low $\left(200-280^{\circ} \mathrm{C}\right)$ and high $\left(>300^{\circ} \mathrm{C}\right)$ synthesis temperatures, respectively (Table 1). This temperature-dependent phase formation preference may be partially attributed to the loss of Dod-SH capping ligand at higher temperatures $\left(>300^{\circ} \mathrm{C}\right)$, which has also been observed for the synthesis of $\mathrm{CuInS}_{2}{ }^{36}$ and $\mathrm{Cu}_{2} \mathrm{FeSnS}_{4}{ }^{37}$ nanocrystals. By replacing Dod$\mathrm{SH}$ with elemental S, pure kesterite CZTS can form at both low $\left(200^{\circ} \mathrm{C}\right)$ and high $\left(300^{\circ} \mathrm{C}\right)$ temperatures with stoichiometric sulfur/ metal ratio, while wurtzite CZTS is formed at $250^{\circ} \mathrm{C}$ for a wide range of sulfur/metal molar ratios (4-32) (Table 1, see Supplementary Fig. S1 online). Elemental S has widely been used for the synthesis of kesterite CZTS nanocrystals ${ }^{9,11,12}$, but we have observed that by using excess sulfur (sulfur/metal molar ratio $=8$ ), wurtzite CZTS can also be successfully obtained at a higher temperature $\left(300^{\circ} \mathrm{C}\right)$ (see Supplementary Fig. S1c online). The excess sulfur likely plays the role of a capping ligand that binds to specific crystal planes and favors the growth of the wurtzite phase. In general, the nature of sulfur source, reaction temperature, sulfur/metal molar ratio are primary factors that influence the crystals phase of CZTS nanocrystals.

The X-ray diffraction (XRD) patterns of typical as-synthesized CZTS nanocrystal samples and crystal structures of wurtzite-type and kesterite-type $\mathrm{Cu}_{2} \mathrm{ZnSnS}_{4}$ are shown in Figure 2. Rietveld refinements (blue line in Figure 2 and Supplementary Table S1-S4) are utilized to further analyze the basic crystal structure parameters of CZTS nanocrystals based on the experimental XRD patterns. Figure 2a shows the experimental (red dots) and simulated XRD pattern (red line) for the wurtzite CZTS nanocrystals. Since no standard XRD pattern belonging to wurtzite CZTS exists in the database, a simulated pattern has been obtained based on the structure. Both the experimental and simulated patterns match well, confirming the

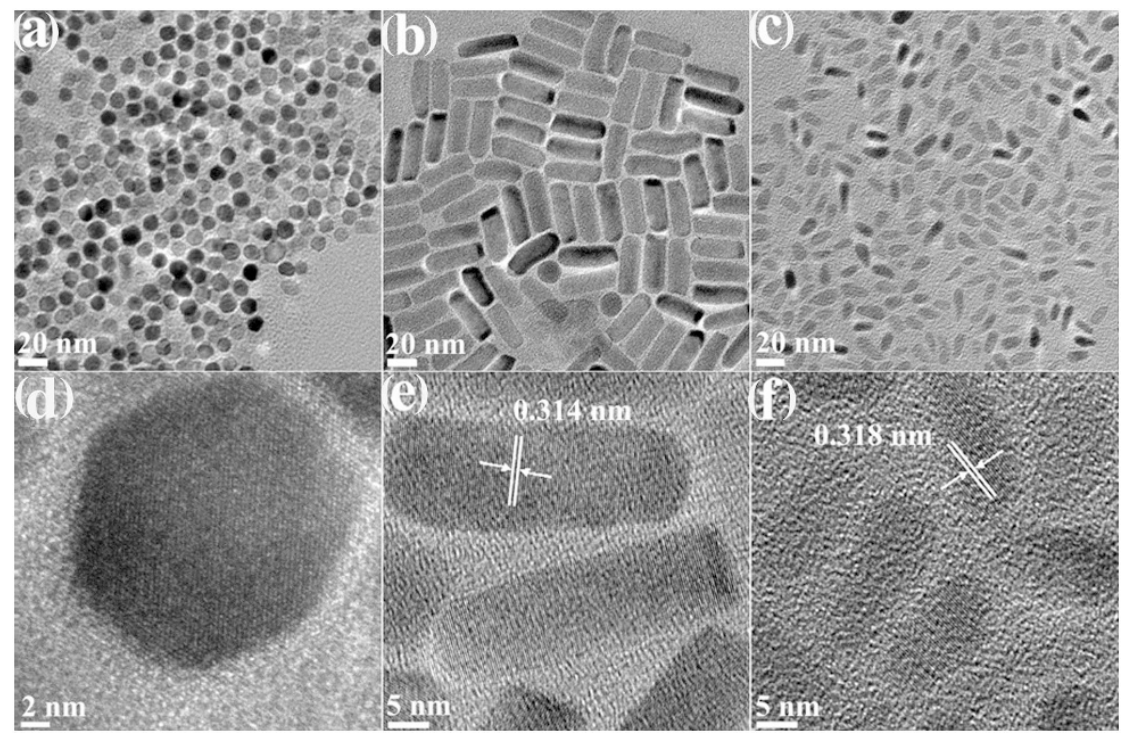

Figure $1 \mid(a-c)$ TEM and (d-f) HRTEM images of wurtzite-structured CZTS nanocrystals with (a,d) spherical, (b,e) rod-like, and (c,f) rice-like shapes. 


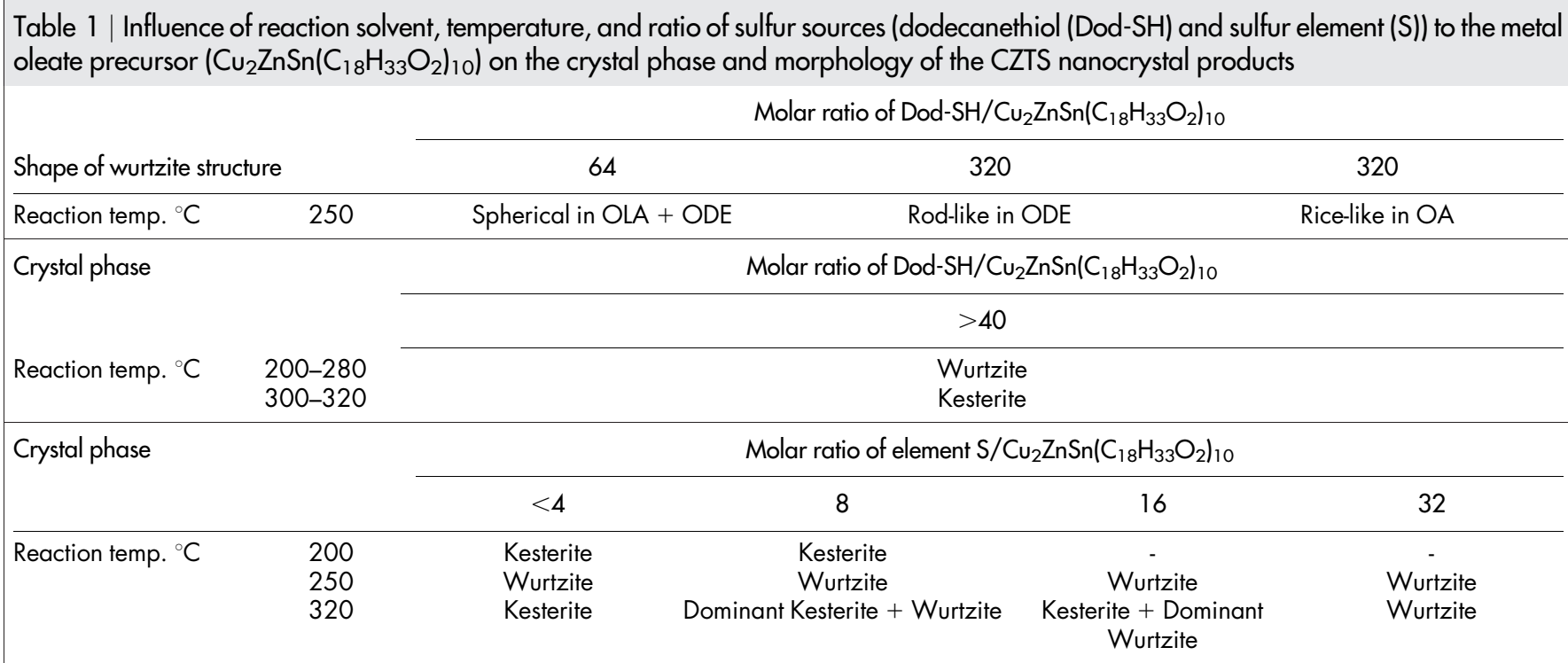

wurtzite structure of the nanocrystals. The crystal structure parameters obtained from Rietveld refinement of the experimental pattern are $a=3.809 \AA$ and $c=6.300 \AA$ with space group of $P 6_{3} m c$. Figure $2 \mathrm{~b}$ shows the experimental (red dots) and standard XRD pattern (red line) of typical Kesterite CZTS nanocrystals. The peak position and relative intensity of the primary diffraction peaks for the nanocrystals agree well with the standard kesterite diffraction pattern (space group $I \overline{4} 2 \mathrm{~m} ; a=5.427 \AA ; c=10.840 \AA$. JCPDS No. 26-0575, Figure $2 \mathrm{~b}$ ). The kesterite and stannite powder XRD patterns differ only slightly in the splitting of the higher order peaks, such as of $(220) /(204)$ and $(116) /(312)$, due to small differences in the tetragonal distortion $(c / 2 a)$. Thus, it is difficult to unambiguously distinguish between the kesterite and stannite structures using XRD alone because of peak broadening, as reported previously ${ }^{12}$. Some of the minor peaks are also not clearly observed because of the small size of the nanocrystals. Similar Rietveld refinements (Figure $2 b$ blue line, and Supplementary Table S3, S4) have been done for kesterite CZTS nanocrystals. The crystal structure parameters obtained from Rietveld refinement of the experimental pattern are $a=5.432 \AA$ and $c=10.764 \AA$ with space group of $I \overline{4} 2 \mathrm{~m}$. No additional peaks belonging to $\mathrm{ZnS}, \mathrm{SnS}$ and $\mathrm{Cu}_{2} \mathrm{~S}$ are observed in the diffraction patterns, which confirm the synthesized CZTS nanocrystals are single phase.
Previous studies have shown that the elemental composition of CZTS nanocrystals strongly influences the performance of the final device. The best CZTS devices have been obtained with $\mathrm{Cu}$-poor and $\mathrm{Zn}$-rich stoichiometry, with $\mathrm{Cu} /(\mathrm{Zn}+\mathrm{Sn})$ ratio in the range of 0.8 to 0.95 , to avoid the formation of impurity binary or ternary copper chalcogenides that can degrade device performance ${ }^{9,38,39}$. However, a recent study on CZTSe nanocrystal solar cells shows that an intermediate $\mathrm{Cu}$-rich precursor together with $\mathrm{KCN}$ treatment for etching the $\mathrm{Cu}_{\mathrm{x}} \mathrm{S}$ impurities results in enhanced cell efficiency of $7.5 \%$, partly because of the better current collection properties ${ }^{40}$. Thus, precise compositional control of quaternary chalcogenide nanocrystals is very important. In our study, the elemental composition of as-synthesized CZTS nanocrystals is readily controlled by simply varying the molar ratio of $\mathrm{Cu} /(\mathrm{Zn}+\mathrm{Sn})$ in the as-prepared $\mathrm{Cu}_{\mathrm{x}} \mathrm{ZnSn}$ $\left(\mathrm{C}_{18} \mathrm{H}_{33} \mathrm{O}_{2}\right)_{6+2 x}$ metal-oleate precursor, with ratios from 0.5 to 1.2 in interval steps of 0.1 . The crystal structure of all the as-synthesized CZTS nanocrystal products under appropriate reaction conditions has been confirmed to be pure wurtzite structure without any other binary or ternary impurities, as confirmed by XRD (see Supplementary Fig. S2 online). This further illustrates the advantage of wurtzite structure for flexible stoichiometry control. The average elemental composition of the synthesized CZTS nanocrystals, as determined by Energy-dispersive X-ray spectroscopy (EDAX), show
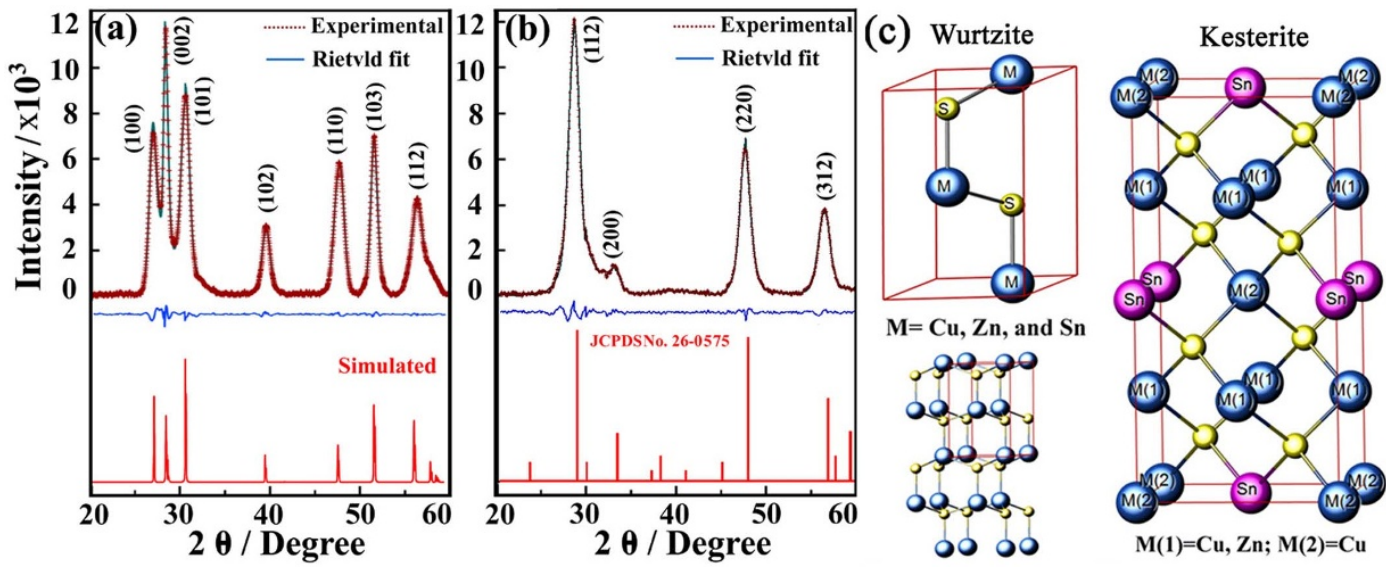

Figure $2 \mid$ (a, b) Experimental (red dots), simulated (red line), and Rietveld fit (blue line) XRD patterns of representative CZTS nanocrystals with (a) wurtzite and (b) kesterite structures. Standard XRD pattern (JCPDS No. 26-0575) of kesterite structured CZTS is also given in Figure 2b. (c) Illustration of crystal structure of unit cell and $2 \times 2 \times 2$ unit cells of wurtzite-type $\mathrm{Cu}_{2} \mathrm{ZnSnS}_{4}$, and unit cell of kesterite -type $\mathrm{Cu}_{2} \mathrm{ZnSnS}_{4}$. 

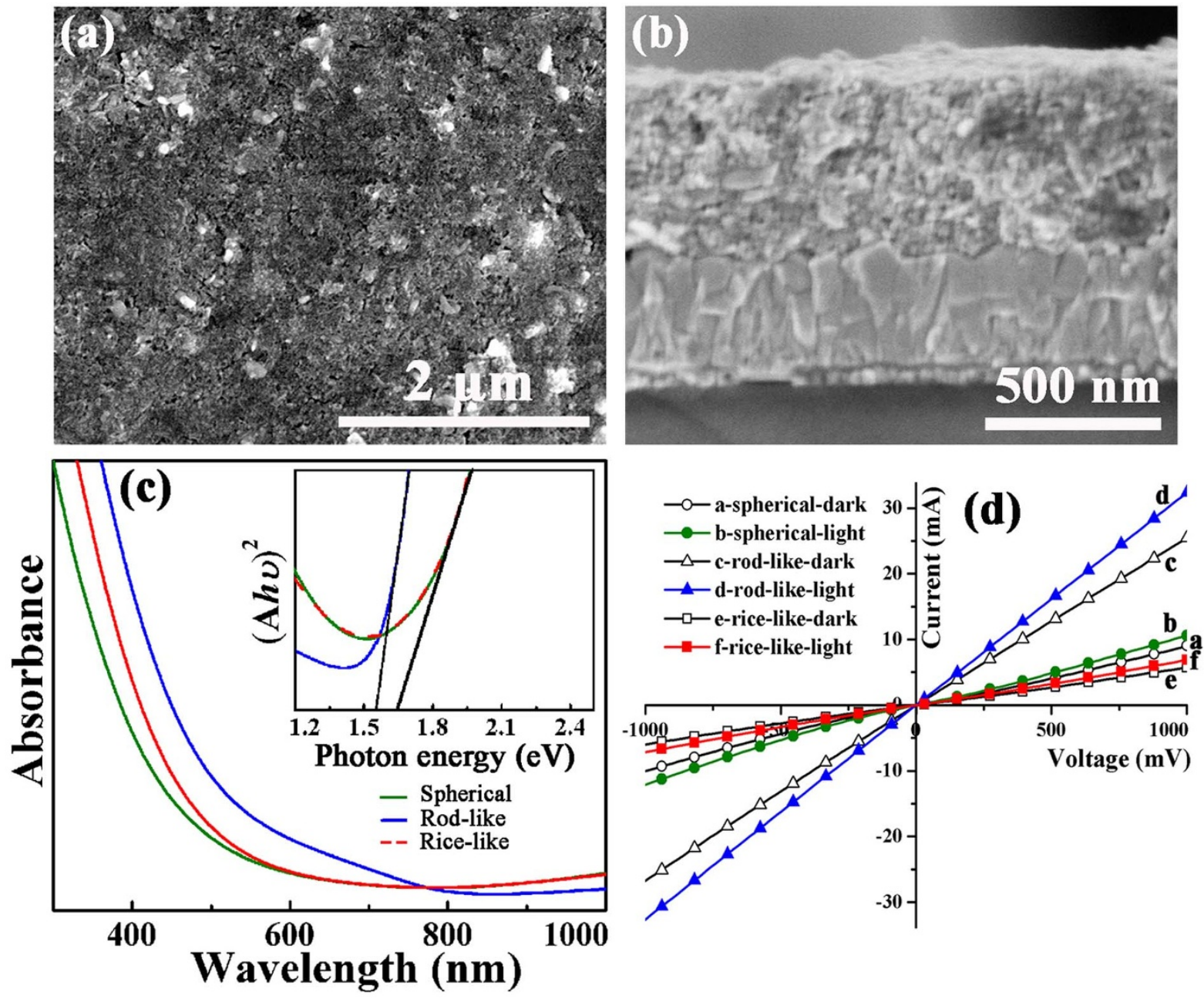

Figure 3 SEM images of (a) top and (b) cross-section view of CZTS thin film fabricated from CZTS nanorods. (c) Room temperature absorption spectra for spherical, rod-like and rice-like CZTS nanocrystals. Inset shows extrapolation of the spectra in the band edge region for determination of the band gap. (d) Photocurrent response of CZTS nanocrystal thin films made from a-b spherical, c-d rod-like, and e-f rice-like CZTS nanocrystals.

the composition is tunable and consistent $\mathrm{Cu} /(\mathrm{Zn}+\mathrm{Sn})$ ratios for the CZTS nanocrystal products (Supplementary Table S5) are observed. Note that CZTS nanocrystals with decreasing copper content show some deviation from the expected elemental ratio, which may be due to the difficulty in forming a stoichiometric solid solution with larger $\mathrm{Sn}$ atoms, similar to that reported for $\mathrm{Cu}-\mathrm{In}-\mathrm{S}$ nanocrys$\operatorname{tals}^{15}$. The optical properties of CZTS nanocrystals with wurtzite and kesterite structures have been studied by UV-Vis absorption spectroscopy (see Supplementary Fig. S3 online). The band gaps have been obtained by plotting $(\mathrm{A} h v)^{2}$ versus $h v(\mathrm{~A}=$ absorbance, $h=$ Planck's constant and $v=$ frequency) and extrapolating the linear portion of the spectrum in the band edge region. The band gaps are determined to be $1.56 \pm 0.04 \mathrm{eV}$ and $1.45 \pm 0.03 \mathrm{eV}$ for the wurtzite and kesterite CZTS nanocrystals, respectively. These values are close to the theoretical energy gap of $\sim 1.5 \mathrm{eV}$ for kesterite $\mathrm{CZTS}^{41}$, and the reported 1.4-1.6 eV values for the kesterite ${ }^{9-12}$ and wurtzite ${ }^{20-22}$ CZTS nanocrystals.

In order to evaluate its potential as an active layer for photovoltaic applications, we have measured the optical, photoconductivity, and electrical properties of wurtzite CZTS nanocrystal films with shape variation. The morphology and thickness of the thin films have been investigated using a Scanning Electron Microscope (SEM). As shown in Figure 3(a,b), the surface of the CZTS nanorod films displays good uniformity over a large area with very few cracks and the cross-section of the film exhibits densely packed structure with thickness of $\sim 500 \mathrm{~nm}$. The optical properties for wurtzite CZTS with spherical, rod-like and rice-like shapes have been investigated using UV-vis absorption spectroscopy (Figure 3c). The band gap for rodlike CZTS nanocrystals has been determined to be around $1.55 \pm$ $0.04 \mathrm{eV}$, which is in accordance with reported values ${ }^{20-22}$. A blue shift to longer wavelength is observed for both spherical and rice-like CZTS nanocrystals, with band gaps of around $1.65 \pm 0.03 \mathrm{eV}$. As compared to rod-like nanocrystals, spherical and rice-like CZTS nanocrystals have smaller particle size of around 10-12 nm (Figure 1a,c). Thus the blue shift may be due to size effect, which has previously also been observed for $\mathrm{CuInS}_{2}$ nanocrystals ${ }^{42}$. The photoresponse properties of the films have been studied with FTO and ITO glass as bottom and top electrode, respectively. I-V curves of thin films after annealing at $300^{\circ} \mathrm{C}$ have been measured both in the dark and under illumination using a solar simulator (AM $1.5 \mathrm{G}$ irradiation, $100 \mathrm{~mW} \cdot \mathrm{cm}^{-2}$ ). A current enhancement is observed under illumination for all samples, as shown in Figure $3 \mathrm{~d}$, which has also been reported for other semiconductor nanocrystal films ${ }^{20}$. The dark current for three samples varies in the sequence rice-like $<$ spherical < rod-like CZTS thin films, whereas the measured photocurrents are higher by $39 \%$ for rice-like, $17.2 \%$ for spherical shape, and $27.1 \%$ for rod-like CZTS thin films at $1000 \mathrm{mV}$ as compared to the dark current. The photoresponse behavior exhibits obvious shape-dependent properties. The comparatively lower photocurrent for spherical shaped and rice-like CZTS nanocrystals may be due to charge recombination at the grain boundaries. In contrast, 1D rodlike CZTS thin films display the highest photocurrent, benefitting 
Table 2 | Electrical properties of wurtzite CZTS NCs films with different morphologies determined using Hall measurement system

\begin{tabular}{|c|c|c|c|}
\hline Sample & Resistivity $\left(10^{3} \Omega \cdot \mathrm{cm}\right)$ & Carrier concentration $\left(10^{12} \mathrm{~cm}^{-3}\right)$ & Hall mobility $\left(\mathrm{cm}^{2} V^{-1} \mathrm{~s}^{-1}\right)$ \\
\hline
\end{tabular}

from improved charge transfer characteristics similar to other $1 \mathrm{D}$ nanostructures, such as $\mathrm{TiO}_{2}$ nanotube and nanowires ${ }^{43,44}$. Besides, the nanorods display better photocurrent behaviour than nanospheres could also be indicative of the inefficiency of removal of oleate coating for the spherical CZTS. The difference of photocurrents between particles with rice and sphere-like shapes is interesting because the rice-like shaped particles likely have a less dense capping layer at the sharp apex and thus facilitate carrier transfer.

Efficiency of the semiconductor NPs as the PV materials is strongly dependent on the possibility to remove the insulating gaps between the NPs in the deposited films ${ }^{45}$, 46 . We have also investigated the shape-dependent variations in terms of electrical conductivity, carrier concentration, and Hall mobility. The electrical properties have been studied by Hall effect measurements with applied magnetic field of $0.56 \mathrm{~T}$ using four point probe with the Van der Pauw method. As determined from the Hall coefficient, all the different shape samples exhibit p-type conductivity. The resistivity, carrier concentrations, and Hall mobility of CZTS for various shapes are summarized in Table 2. The CZTS thin film composed of rod-like nanocrystals exhibits the lowest resistivity, which may be due to efficient charge transfer for 1-D structure, and this is consistent with the high current observed in the I-V curve (Figure 3d). Furthermore, the rod-like CZTS display the highest carrier concentration as compared to the spherical and rice-like nanocrystals, which is beneficial for photovoltaic application. As for the Hall mobility, spherical and rice-like NCs show similar values, and are higher than that for the rod-like sample.

The preliminary results based on photo-response behavior demonstrate that CZTS nanocrystals have potential for use as the active layer in thin film solar cells. 1D nanostructures with tunable geometric parameters, such as length and diameter, can allow to maximize the optical absorption and improve charge transfer characteristics. Their electrical properties satisfy the requirements for potential application in thin film photovoltaic cells.

\section{Discussion}

Metal-oleate complexes formed via low-temperature reaction of the constituent metal halides with sodium oleate have been utilized for the thermolysis synthesis of a variety of monodisperse oxides ${ }^{28-31}$. The synthesis of chalcogenide nanocrystals using metal-oleate complexes has thus far been limited to copper-indium sulfide nanocrystal heterostructures ${ }^{13,35}$. Instead of forming nanocrystals of uniform composition, segregation of $\mathrm{Cu}_{2} \mathrm{~S} / \mathrm{In}_{2} \mathrm{~S}_{3}$ and $\mathrm{Cu}_{2} \mathrm{~S} / \mathrm{CIS}$ results in the formation of unusual heterostructures of various shapes. This behavior can primarily be attributed to the differences in the decomposition temperature of the $\mathrm{Cu}$ - and In-oleate precursors. In the present study, the homogeneous nucleation and growth of CZTS nanocrystals with well-controlled morphologies are due to the use of a single source precursor of $\mathrm{Cu}_{2} \mathrm{ZnSn}$-oleate complexes. The TGA curves (Figure 4a) for the $\mathrm{Cu}_{2}\left(\mathrm{C}_{18} \mathrm{H}_{33} \mathrm{O}_{2}\right)_{4}$ and $\mathrm{Sn}\left(\mathrm{C}_{18} \mathrm{H}_{33} \mathrm{O}_{2}\right)_{4}$ complexes exhibit similar weight loss behavior over a close temperature range, while the TGA curve of the $\mathrm{Zn}\left(\mathrm{C}_{18} \mathrm{H}_{33} \mathrm{O}_{2}\right)_{2}$ complex shows weight loss at higher temperatures. In contrast, the TGA curve of the mixed $\mathrm{Cu}_{2} \mathrm{ZnSn}$-oleate complexes shows a small weight loss below $200^{\circ} \mathrm{C}$ and a much large, rapid and continuous weight loss at temperatures above $250^{\circ} \mathrm{C}$, indicating that the $\mathrm{Cu}_{2} \mathrm{ZnSn}$-oleate complex is intimately mixed, essentially forming a single precursor of $\mathrm{Cu}_{2} \mathrm{ZnSn}\left(\mathrm{C}_{18} \mathrm{H}_{33} \mathrm{O}_{2}\right)_{10}$ complex with similar decomposition temperatures. This is critical for the compositional and structural uniformity of the nanocrystals. This has also been conclusively demonstrated in our earlier study of the synthesis of ferrites using well-mixed metal-oleate complexes as precursors ${ }^{31}$. Besides the TGA curves, the DSC curves for $\mathrm{Cu}_{2}\left(\mathrm{C}_{18} \mathrm{H}_{33} \mathrm{O}_{2}\right)_{4}$, $\mathrm{Sn}\left(\mathrm{C}_{18} \mathrm{H}_{33} \mathrm{O}_{2}\right){ }_{4}, \mathrm{Zn}\left(\mathrm{C}_{18} \mathrm{H}_{33} \mathrm{O}_{2}\right)_{2}$ and $\mathrm{Cu}_{2} \mathrm{ZnSn}\left(\mathrm{C}_{18} \mathrm{H}_{33} \mathrm{O}_{2}\right)_{10}$ complexes have been measured as shown in Figure $4 \mathrm{~b}$ that provide useful information regarding the thermal changes of the complexes during the heating-up process. An endothermic peak below $100^{\circ} \mathrm{C}$ indicates the existence of small amount of water in the $\mathrm{Zn}\left(\mathrm{C}_{18} \mathrm{H}_{33} \mathrm{O}_{2}\right)_{2}$ complex due to its propensity for hydrolysis in air. The temperature for the weight losses (Figure 4a) matches well with that for the thermal changes (Figure $4 \mathrm{~b}$ ), which further confirms the formation of a single precursor of $\mathrm{Cu}_{2} \mathrm{ZnSn}\left(\mathrm{C}_{18} \mathrm{H}_{33} \mathrm{O}_{2}\right)_{10}$ complex.

To better understand the formation of $1 \mathrm{D}$ rod-like and rice-like CZTS nanocrystals, the nucleation and growth processes for each shape have been systematically studied. As seen in Figure 5, by merely changing the aging time, along with increasing the size of nanocrystals the shape of the nanocrystals varies from spherical nanodots at $0.5 \mathrm{~min}$ (Figure 5a), to elongated nanocrystals at $1.5 \mathrm{~min}$ (Figure 5b), mixed short nanorods at $5 \mathrm{~min}$ (Figure 5c), and nanorods at $10 \mathrm{~min}$ (Figure $5 \mathrm{~d}$ ). The final products formed at $30 \mathrm{~min}$ are uniform nanorods (Figure $1 \mathrm{~b}$ ) having the wurtzite structure (see Supplementary Fig. S4a online). In contrast, the formation
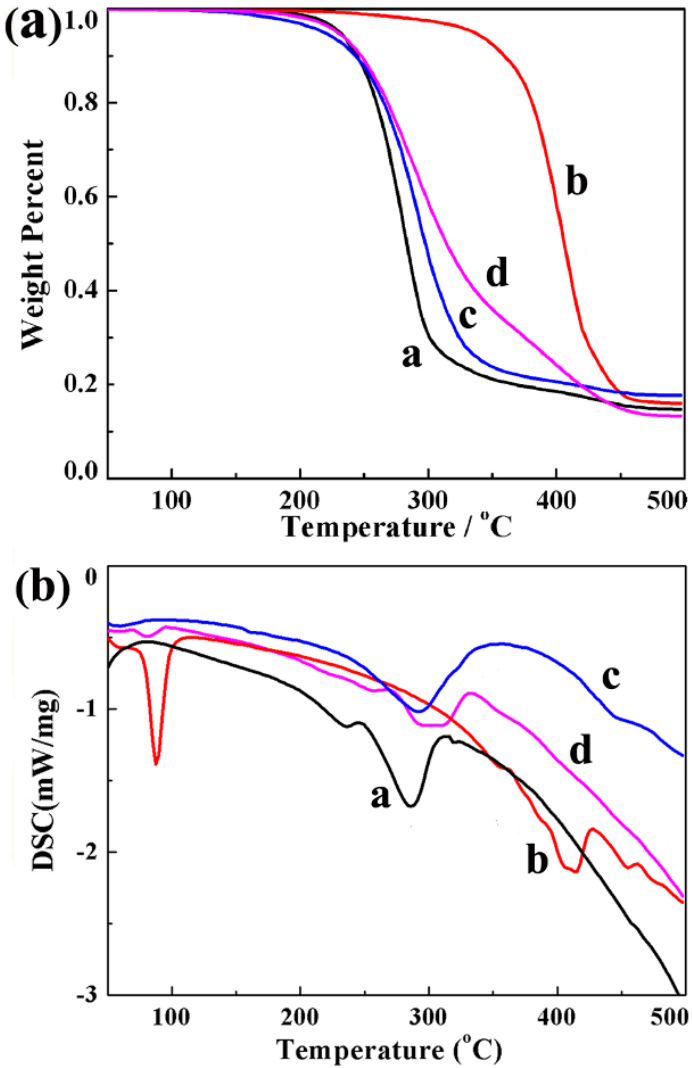

Figure $4 \mid$ (a) TGA and (b) DSC curves of a. $\mathrm{Cu}_{2}\left(\mathrm{C}_{18} \mathrm{H}_{33} \mathrm{O}_{2}\right)_{4}$, b. $\mathrm{Zn}\left(\mathrm{C}_{18} \mathrm{H}_{33} \mathrm{O}_{2}\right)_{2}, \mathrm{c} . \mathrm{Sn}\left(\mathrm{C}_{18} \mathrm{H}_{33} \mathrm{O}_{2}\right)_{4}$, and d mixed $\mathrm{Cu}_{2} \mathrm{ZnSn}\left(\mathrm{C}_{18} \mathrm{H}_{33} \mathrm{O}_{2}\right)_{10}$ oleate complexes. 


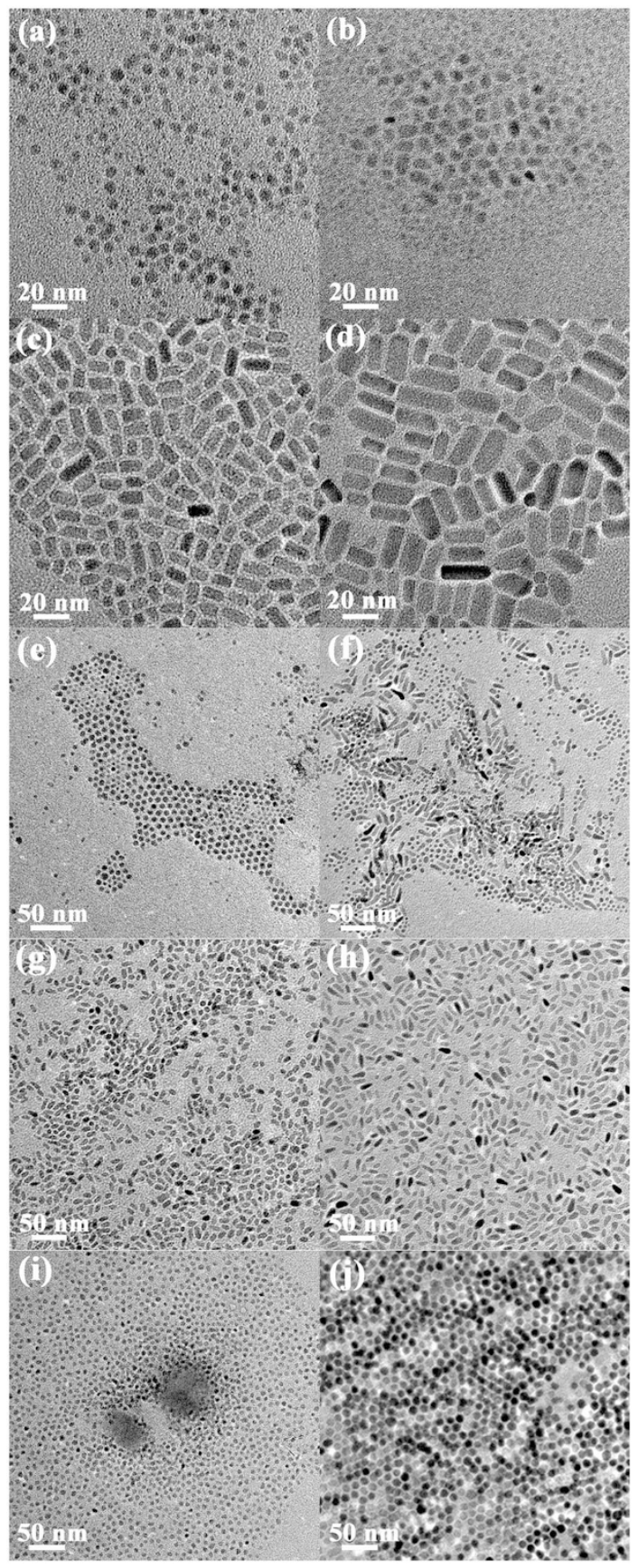

Figure 5 TEM images of CZTS nanocrystals sampled from the reaction solution at $250^{\circ} \mathrm{C}$ after aging for $(\mathrm{a}, \mathrm{e}, \mathrm{i}) \mathbf{0 . 5},(\mathrm{b}, \mathrm{f}, \mathrm{j}) \mathbf{1 . 5},(\mathrm{c}, \mathrm{g}) 5$, and $(\mathrm{d}, \mathrm{h})$ $10 \mathrm{~min}$ showing the formation of rod-like $(\mathrm{a}-\mathrm{d})$, rice-like $(\mathrm{e}-\mathrm{h})$, and spherical $(\mathrm{i}, \mathrm{j})$ CZTS nanocrystals after aging for $30 \mathrm{~min}$.

of rice-like nanocrystals involves initial formation of spherical nanodots at $0.5 \mathrm{~min}$ (Figure 5e), dominant spherical nanodots mixed with a few long tadpole-like nanocrystals at $1.5 \mathrm{~min}$ (Figure $5 \mathrm{f}$ ), and ricelike shapes with increased size after 5 and $10 \mathrm{~min}$ (Figure $5 \mathrm{~g}-\mathrm{h}$ ). Further aging for $20 \mathrm{~min}$ results in the formation of uniform ricelike nanocrystals (Figure 1c) with the wurtzite structure (see Supplementary Fig. S4b online).

The shape of the nanocrystal products is found to be strongly influenced by the precursor concentration and nature of solvents $s^{47-50}$. At high precursor concentrations, many nuclei with uniform small size (Figure 5i) are formed abruptly because of the rapid decomposition of most of the precursor. All the nuclei then grow uniformly at a high rate until the residual precursor is consumed, eventually forming monodisperse spherical nanocrystals (Figure 1a). In contrast, low precursor concentration in neutral ODE or acidic OA allows for relatively slow nucleation, with most of the precursor still present in the solvent. Thus, larger nuclei grow at the expense of smaller nuclei (Figure 5a-b, e-f). The relatively slow growth rate of larger nuclei results in the formation of metastable 1D nanostructures, such as rod-like (Figure $5 \mathrm{c}-\mathrm{d}$ ), tadpole-like, and rice-like shapes (Figure $5 \mathrm{~g}-\mathrm{h}$ ). It is noted that a few tadpole-like nanocrystal intermediate with relatively large size (Figure $5 f$ ) initially formed are then converted into uniform small size rice-like nanocrystals with prolonged reaction time. This process is somewhat different from the well-known interparticle Ostwald ripening process, which occurs at monomer concentrations several times lower than for intrerparticle ripening ${ }^{49}$. On the other hand, the etching effect of free OA, which can dissolve the relatively small nanocrystals, and balance between the process of growth and dissolution of nanocrystals, will further influence the morphology evolution during crystal growth ${ }^{50}$. The wurtzite structure of CZTS nanocrystals that favors the growth of anisotropic rod-like and rice-like shapes has also been observed for the synthesis of other wurtzite-structured semiconductors, such as CIGS, $\mathrm{ZnO}$, etc ${ }^{16,51,52}$.

In conclusion, we have reported a solution-based method for the synthesis of CZTS nanocrystals by hot-injection of a single source mixed metal-oleate complex into hot organic solvents with dissolved sulfur sources. Depending on the reaction conditions, monodisperse CZTS nanocrystals with both wurtzite and kesterite crystal structures can be synthesized with spherical, rice-like, and rod-like shapes. The band gaps for CZTS nanocrystals with wurtzite and kesterite structures are determined to be 1.56 and $1.45 \mathrm{eV}$, respectively. The composition of the as-synthesized CZTS nanocrystals can be readily controlled over a wide range $(0.5 \leq \mathrm{Cu} /(\mathrm{Zn}+\mathrm{Sn}) \leq 1.2)$ by merely changing the molar ratio of cations in the precursor. The synthesized CZTS nanocrystals, showing obvious shape-dependent photoresponse behavior, with highest obtained photocurrent for rod-like CZTS nanocrystals, are promising as light absorber materials.

\section{Methods}

Preparation of mixed metal-oleate complexes $\mathrm{Cu}_{2} \mathrm{ZnSn}-\left(\mathrm{C}_{18} \mathrm{H}_{33} \mathrm{O}_{2}\right)_{10}$. For the preparation of metal-oleate complexes, $20 \mathrm{mmol}$ sodium oleate and a mixture of $4 \mathrm{mmol} \mathrm{Cu}^{2+}, 2 \mathrm{mmol} \mathrm{Zn}^{2+}$, and $2 \mathrm{mmol} \mathrm{Sn}^{4+}$ chlorides were dissolved in mixed solvents of $20 \mathrm{ml} \mathrm{H}_{2} \mathrm{O}, 15 \mathrm{ml}$ ethanol, and $30 \mathrm{ml}$ hexane at $60^{\circ} \mathrm{C}$ under reflux for $4 \mathrm{~h}$. The resulting metal-oleate complex $\mathrm{Cu}_{2} \mathrm{ZnSn}-\left(\mathrm{C}_{18} \mathrm{H}_{33} \mathrm{O}_{2}\right)_{10}$ was obtained by separation of the water phase and subsequent evaporation of the residual ethanol and hexane at $70^{\circ} \mathrm{C}$ and water at $110^{\circ} \mathrm{C}$.

Synthesis of $\mathrm{Cu}_{2} \mathrm{ZnSnS}_{4}$ nanocrystals. All the experiments were carried out in a fume hood under inert $\mathrm{N}_{2}$ atmosphere using standard Schlenk techniques. In a typical synthesis of spherical nanocrystals, $2 \mathrm{~g} \mathrm{Cu}_{2} \mathrm{ZnSn}-\left(\mathrm{C}_{18} \mathrm{H}_{33} \mathrm{O}_{2}\right)_{10}$ was dissolved in $2 \mathrm{ml}$ $\mathrm{ODE}$ at $120^{\circ} \mathrm{C}$ under starring, forming a homogeneous precursor solution. $5 \mathrm{ml}$ Dod$\mathrm{SH}$ was dissolved in a mixture of $10 \mathrm{ml}$ OLA and $15 \mathrm{ml}$ ODE in a four-neck roundbottom flask and stirred at room temperature for $30 \mathrm{~min}$ with nitrogen purging. The solution was then heated to $250^{\circ} \mathrm{C}$, and $2 \mathrm{ml}$ precursor solution was rapidly injected into the solution under a $\mathrm{N}_{2}$ atmosphere with continuous stirring. The reaction solution was maintained at $250^{\circ} \mathrm{C}$ for $30 \mathrm{~min}$, and was subsequently cooled down to room temperature. A mixture of hexane and ethanol was used to precipitate the product and collect it via centrifugation. Rod and rice-shaped nanocrystals were synthesized by injecting $0.4 \mathrm{ml}$ precursor solution in $25 \mathrm{ml} \mathrm{ODE}$ and $25 \mathrm{ml} \mathrm{OA}$, respectively. The other procedures were the same as described for the synthesis of spherical nanocrystals.

Deposition of $\mathrm{Cu}_{2} \mathrm{ZnSnS}_{4}$ thin films. The $\mathrm{Cu}_{2} \mathrm{ZnSnS}_{4}$ thin films were deposited by a spray-painting method. Firstly, the $\mathrm{Cu}_{2} \mathrm{ZnSnS}_{4}$ nanocrystals with different shapes were dispersed in ethanol to form an ink with concentration of $\sim 2.5-5 \mathrm{mg} / \mathrm{ml}$. $\mathrm{Cu}_{2} \mathrm{ZnSnS}_{4}$ thin films were spray-deposited using the suspension onto FTO glass, followed by annealing under $\mathrm{N}_{2}$ atmosphere at $300^{\circ} \mathrm{C}$ for $30 \mathrm{~min}$. The photoresponsive properties of the NCs have been studied with FTO and ITO glass as bottom and top electrode, respectively.

Characterization. The morphology and size distribution of the nanocrystals were studied using transmission electron microscopy (TEM, FEI Tecnai F-20). X-ray powder diffraction (XRD, Bruker D8) was used to study the structure of the nanocrystals. The optical properties were investigated using a UV-Vis spectrometer (Varian Cary 50). The composition of the nanocrystals was determined using energy- 
dispersive $\mathrm{x}$-ray spectroscopy (EDAX). Thermogravimetric analyses (TGA) were performed on a NETZSCH STA 449C thermogravimetric analyzer. For determining the photoresponse behaviour, a solar simulator with an illumination intensity of one sun (AM 1.5, $100 \mathrm{~mW} / \mathrm{cm}^{2}$, Newport Corporation) was used as the light source for illumination. An EG\&G potentiostat was used to measure the photoresponse of the thin films. The carrier concentration, Hall mobility, and electrical resistivity were obtained using a Hall effect measurement system (HMS-3000, Ecopia) with applied magnetic field of $0.56 \mathrm{~T}$ by a four point probe with the Van der Pauw method.

1. Habas, S. E., Platt, H. A. S., van Hest, M. F. A. M. \& Ginley, D. S. Low-cost inorganic solar cells: from ink to printed device. Chem. Rev. 110, 6571-6594 (2010).

2. Ip, A. H. et al. Hybrid passivated colloidal quantum dot solids. Nature Nanotech. 7, 577-582 (2012).

3. Koleilat, G. I. et al. Folded-light-path colloidal quantum dot solar cells. Sci. Rep. 3 2166 (2013)

4. El-Sayed, M. A. Small is different: shape-, size-, and composition-dependent properties of some colloidal semiconductor nanocrystals. Acc. Chem. Res. 37, 326-333 (2004).

5. Xia, Y., Xiong, Y., Lim, B. \& Skrabalak, S. E. Shape-controlled synthesis of metal nanocrystals: simple chemistry meets complex physics? Angew. Chem. Int. Ed. 48, 60 (2009).

6. Kongkanand, A., Tvrdy, K., Takechi, K., Kuno, M. \& Kamat, P. V. Quantum dot solar cells. tuning photoresponse through size and shape control of $\mathrm{CdSe}-\mathrm{TiO}_{2}$ architecture. J. Am. Chem. Soc. 130, 4007-4015 (2008).

7. Wang, W. et al. Device characteristics of CZTSSe thin-film solar cells with $12.6 \%$ efficiency. Adv. Energy. Mater. 4, 1301465 (2014).

8. Woo, K. et al. Band-gap-graded $\mathrm{Cu}_{2} \mathrm{ZnSn}\left(\mathrm{S}_{1-\mathrm{x}}, \mathrm{Se}_{\mathrm{x}}\right)_{4}$ solar cells fabricated by an ethanol-based, particulate precursor ink route. Sci. Rep. 3, 3069 (2013)

9. Guo, Q. et al. Fabrication of 7.2\% efficient CZTSSe solar cells using CZTS nanocrystals. J. Am. Chem. Soc. 132, 17384-17386 (2010).

10. Cao, Y. et al. High-efficiency solution-processed $\mathrm{Cu}_{2} \mathrm{ZnSn}(\mathrm{S}, \mathrm{Se})_{4}$ thin-film solar cells prepared from binary and ternary nanoparticles. J. Am. Chem. Soc. 134, 15644-15647 (2012)

11. Steinhagen, C., Panthani, M. G., Akhavan, V., Goodfellow, B., Koo, B. \& Korgel, B. A. Synthesis of $\mathrm{Cu}_{2} \mathrm{ZnSnS}_{4}$ nanocrystals for use in low-cost photovoltaics. J. Am. Chem. Soc. 131, 12554-12555 (2009).

12. Guo, Q., Hillhouse, H. W. \& Agrawal, R. Synthesis of $\mathrm{Cu}_{2} \mathrm{ZnSnS}_{4}$ nanocrystal ink and its use for solar cells. J. Am. Chem. Soc. 131, 11672-11673 (2009).

13. Conner, S. T., Hsu, C., Weil, B. D., Aloni, S. \& Cui, Y. Phase transformation of biphasic $\mathrm{Cu}_{2} \mathrm{~S}-\mathrm{CuInS} \mathrm{H}_{2}$ to monophasic $\mathrm{CuInS}_{2}$ nanorods. J. Am. Chem. Soc. 131, 4962-4966 (2009).

14. Qi, Y., Liu, Q., Tang, K., Liang, Z., Ren, Z. \& Liu, X. Synthesis and characterization of nanostructured wurtzite $\mathrm{CuInS}_{2}$ : a new cation disordered polymorph of CuInS 2 . J. Phys. Chem. C 113, 3939-3944 (2009).

15. Pan, D. et al. Synthesis of Cu-In-S ternary nanocrystals with tunable structure and composition. J. Am. Chem. Soc. 130, 5620-5621 (2008).

16. Wang, Y., Zhang, X., Bao, N., Lin, B. \& Gupta, A. Synthesis of shape-controlled monodisperse wurtzite $\mathrm{CuIn}_{\mathrm{x}} \mathrm{Ga}_{1-\mathrm{x}} \mathrm{S}_{2}$ semiconductor nanocrystals with tunable band gap. J. Am. Chem. Soc. 133, 11072-11075 (2011).

17. Norako, M. E., Greaney, M. J. \& Brutchey, R. L. Synthesis and characterization of wurtzite-phase copper tin selenide nanocrystals. J. Am. Chem. Soc. 134, 23-26 (2012).

18. Kumar, P., Uma, S. \& Nagarajan, R. Precursor driven one pot synthesis of wurtzite and chalcopyrite $\mathrm{CuFeS}_{2}$. Chem. Commun. 49, 7316-7318 (2013).

19. Cui, Y., Wang, G. \& Pan D. Synthesis and photoresponse of novel Cu2CdSnS4 semiconductor nanorods. J. Mater. Chem. 22, 12471-12473 (2012).

20. Lu, X., Zhuang, Z., Peng, Q. \& Li, Y. Wurtzite $\mathrm{Cu}_{2} \mathrm{ZnSnS}_{4}$ nanocrystals: a novel quaternary semiconductor. Chem. Commun. 47, 3141-3143 (2011).

21. Singh, A., Geaney, H., Laffir, F. \& Ryan, K. M. Colloidal synthesis of wurtzite $\mathrm{Cu}_{2} \mathrm{ZnSnS}_{4}$ nanorods and their perpendicular assembly. J. Am. Chem. Soc. 134, 2910-2913 (2012).

22. Li, M. et al. Synthesis of pure metastable wurtzite CZTS nanocrystals by facile onepot method. J. Phys. Chem. C 116, 26507-26516 (2012).

23. Coughlan, C., Singh, A. \& Ryan, K. M. Systematic study into the synthesis and shape development in colloidal $\mathrm{CuIn}_{\mathrm{x}} \mathrm{Ga}_{1-\mathrm{x}} \mathrm{S}_{2}$ nanocrystals. Chem. Mater. 25, 653-661 (2013).

24. Wang, Y., Bao, N., Shen, L., Padhan, P. \& Gupta, A. Size-controlled synthesis of magnetic $\mathrm{CuCr}_{2} \mathrm{Se}_{4}$ nanocrystals. J. Am. Chem. Soc. 129, 12408 (2007).

25. Cao, Y. C. \& Wang, J. One-pot synthesis of high-quality zinc-blende CdS nanocrystals. J. Am. Chem. Soc. 126, 14336-14337 (2004).

26. Pradhan, N. \& Peng, X. Efficient and color-tunable Mn-doped ZnSe nanocrystal emitters: control of optical performance via greener synthetic chemistry. J. Am. Chem. Soc. 129, 3339-3347 (2007)

27. Xie, R., Rutherford, M. \& Peng, X. Formation of high-quality I-III-VI semiconductor nanocrystals by tuning relative reactivity of cationic precursors. J. Am. Chem. Soc. 131, 5691-5697 (2009).

28. An, K. et al. Synthesis, characterization, and self-assembly of pencil-shaped $\mathrm{CoO}$ nanorods. J. Am. Chem. Soc. 128, 9753-9760 (2006).

29. Kwon, S. G. et al. Kinetics of monodisperse iron oxide nanocrystal formation by "heating-up" process. J. Am. Chem. Soc. 129, 12571-12584 (2007).
30. Chen, C., Chen, D., Jiao, X. \& Chen, S. $\operatorname{In}_{2} \mathrm{O}_{3}$ nanocrystals with a tunable size in the range of 4-10 nm: one-step synthesis, characterization, and optical properties. J. Phys. Chem. C 111, 18039-18043 (2007)

31. Bao, N., Shen, L., Wang, Y., Padhan, P. \& Gupta A. A facile thermolysis route to monodisperse ferrite nanocrystals. J. Am. Chem. Soc. 129, 12374-12375 (2007).

32. Choi, S. Y., An, K., Kim, E. G., Yu, J. H., Kim, J. H. \& Hyeon, T. Simple and generalized synthesis of semiconducting metal sulfide nanocrystals. Adv. Funct. Mater. 19, 1645-1649 (2009).

33. Zhong, H. et al. Monodispersed ZnSe colloidal microspheres: preparation, characterization, and their 2D arrays. Langmuir 23, 9008-9013 (2007).

34. Bao, N. et al. Facile thermolysis synthesis of $\mathrm{CuInS}_{2}$ nanocrystals with tunable anisotropic shape and structure. Chem. Commun. 47, 9441-9443 (2011).

35. Choi, S.-H., Kim, E.-G. \& Hyeon, T. One-pot synthesis of copper-indium sulfide nanocrystal heterostructures with acorn, bottle, and larva shapes. J. Am. Chem. Soc. 128, 2520-2521 (2006)

36. Batabyal, S. K., Tian, L., Venkatram, N., Ji, W. \& Vittal, J. J. Phase-selective synthesis of CuInS 2 nanocrystals. J. Phys. Chem. C 113, 15037-15042 (2009).

37. Zhang, X. et al. Crystal phase-controlled synthesis of $\mathrm{Cu}_{2} \mathrm{FeSnS}_{4}$ nanocrystals with a band gap of around $1.5 \mathrm{eV}$. Chem. Commun. 48, 4956-4958 (2012).

38. Todorov, T. K., Reuter, K. B. \& Mitzi, D. B. High-efficiency solar cell with earthabundant liquid-processed absorber. Adv. Mater. 22, E156-E159 (2010).

39. Fairbrother, A. et al. Development of a selective chemical etch to improve the conversion efficiency of $\mathrm{Zn}$-rich $\mathrm{Cu}_{2} \mathrm{ZnSnS}_{4}$ solar cells. J. Am. Chem. Soc. 134, 8018-8021 (2012).

40. Mousel, M. et al. Cu-rich precursors improve kesterite solar cells. Adv. Energy Mater. 4, 1300543 (2014)

41. Persson, C. Electronic and optical properties of $\mathrm{Cu}_{2} \mathrm{ZnSnS}_{4}$ and $\mathrm{Cu}_{2} \mathrm{ZnSnSe}_{4}$. J. Appl. Phys. 107, 053710-1-053710-8 (2010).

42. Lu, X., Zhuang, Z., Peng, Q. \& Li, Y. Controlled synthesis of wurtzite CuInS nanocrystals and their side-by-side nanorod assemblies. Cryst. Eng. Comm. 13 4039-4045 (2011).

43. Feng, X., Zhu, K., Frank, A. J., Grimes, C. A. \& Mallouk, T. E. Rapid charge transport in dye-sensitized solar cells made from vertically aligned single-crystal rutile $\mathrm{TiO}_{2}$ nanowires. Angew. Chem. Int. Ed. 51, 2727-2730 (2012).

44. Varghese, O. K., Paulose, M. \& Grimes, C. A. Long vertically aligned titania nanotubes on transparent conducting oxide for highly efficient solar cells. Nat. Nanotechnol. 4, 592-597 (2009).

45. Tang, J. et al. Colloidal-quantum-dot photovoltaics using atomic-ligand passivation. Nat. Mater. 10, 765-771 (2011)

46. Kim, J.-Y. \& Kotov, N. A. Charge transport dilemma of solution-processed nanomaterials. Chem. Mater. 26, 134-152 (2014).

47. Yin, Y. \& Alivisatos, A. P. Colloidal nanocrystal synthesis and the organicinorganic interface. Nature 437, 664-670 (2005).

48. Lee, S.-M., Cho, S.-N. \& Cheon. Anisotropic shape control of colloidal inorganic nanocrystals. Adv. Mater. 15, 441-444 (2003)

49. Peng, Z. A. \& Peng X. Mechanisms of the shape evolution of CdSe nanocrystals. J. Am. Chem. Soc. 123, 1389-1395 (2001).

50. Zhang, Y., Zhu, J., Song, X. \& Zhong, X. Controlling the synthesis of CoO nanocrystals with various morphologies. J. Phys. Chem. C 112, 5322-5327 (2008)

51. Wang, Z. L. Zinc oxide nanostructures: growth, properties and applications. J. Phys.: Condens. Matter. 16, R829-R858 (2004).

52. Yang, M., Sun, K. \& Kotov, N. A. Formation and assembly-disassembly processes of $\mathrm{ZnO}$ hexagonal pyramids driven by dipolar and excluded volume interactions. J. Am. Chem. Soc. 132, 1860-1872 (2010).

\section{Acknowledgments}

This research was supported by the Natural Science Foundation of China (No. 21006044 No. 51202110), the Natural Science Foundation of Jiangsu Province (No. BK2012426, No BK2012041), the Key Laboratory of Material-Oriented Chemical Engineering of China under Grant No. KL10-02, and the Project of Priority Academic Program Development of Jiangsu Higher Education Institutions (PAPD).

\section{Author contributions}

N.B. contributed to the conception and design of the experiment, analysis of data, and writing the manuscript with the assistance of X.Z. G.G. and C.J. carried out synthesis of materials, optical and structural characterization of products. X.Z., C.J., C.Z. and H.K. performed the photo-response investigation. Y.W. carried out the Rietveld fit and the crystal structure simulation. C.J. carried out the Hall effect measurement, and A.G., L.S. and Y.W. contributed to the analysis of data and modification of the paper. X.Z. and G.G. contributed equally to this work. All authors discussed the results and commented on the manuscript.

\section{Additional information}

Supplementary information accompanies this paper at http://www.nature.com/ scientificreports

Competing financial interests: The authors declare no competing financial interests. 
How to cite this article: Zhang, X.Y. et al. Efficient Thermolysis Route to Monodisperse $\mathrm{Cu}_{2} \mathrm{ZnSnS}_{4}$ Nanocrystals with Controlled Shape and Structure. Sci. Rep. 4, 5086; DOI:10.1038/srep05086 (2014)

(c) (i) (-) This work is licensed under a Creative Commons Attribution-NonCommercialcc. article's Creative Commons license, unless indicated otherwise in the image credit; if the image is not included under the Creative Commons license, users will need to obtain permission from the license holder in order to reproduce the image. To view a copy of this license, visit http://creativecommons.org/licenses/by-nc-sa/3.0/ 